\title{
Studierende aus Russland an der Technischen Hochschule Wildau - Lernsituation und Anpassung an das Lernumfeld ${ }_{[1]}$
}

\author{
Olga Rösch, Bettina Strewe
}

Zusammenfassung

Der vorliegende Artikel hat einige Bereiche der Lernsituation Studierender aus Russland an der Technischen Hochschule Wildau [FH] (University of Applied Sciences) zum Thema. Es geht hierbei hauptsächlich um die Bewertung des Studienaufenthalts und der Lernsituation aus der Perspektive von Studierenden aus Russland, darunter auch von Austauschstudierenden. Die Ausführungen stützen sich auf eine kleine Umfragestudie, die unter der o.g. Zielgruppe an der TH Wildau [FH] durchgeführt wurde.
Abstract

The present article deals with the learning situation of students from Russia presently studying at University of Applied Sciences Wildau as regular or guest students. The focus is mainly on how students from Russia value the study period resp. study visit in Wildau concerning learning situation and learning context. The results are based on a small survey that was carried out among the above mentioned target group.

\section{Einige themenrelevante Daten}

Die Technische Hochschule Wildau [FH] - i. f. TH Wildau genannt - ist eine seit 1991 [2] bestehende Hochschule südöstlich von Berlin mit 23 Studiengängen im Direktstudium und sechs Fernstudiengängen in technischer und verwaltungstechnischer Ausrichtung (http://www.th-wildau.de/vor-dem-studium/hoch schule/fakten.html - 24.06.2010). Den größten Fachbereich stellt das Ingenieurwesen/Wirtschaftsingenieurwesen mit mehreren technisch orientierten Studiengängen. Die Gesamtstudierendenzahl betrug nach Angaben der TH zu Beginn des Wintersemesters 2009 10.3954 Studierende.

\subsection{Ausländische Studierende in Wildau}

Die TH Wildau legt auf die Kooperation mit ausländischen Hochschulen großen Wert. Dies spiegelt sich deutlich in der stetig steigenden Anzahl ausländischer Studierender an der TH Wildau.[3] Die Kontakte zu Hochschulen im Ausland beziehen sich dabei aus der Tradition heraus zum einen überwiegend auf Ost- und Südosteuropa, haben sich allerdings in den letzten Jahren auch auf Lateinamerika und den arabischen Raum erweitert. Es besteht eine Zusammenarbeit mit etwa 20 ausländischen Hochschulen. Viele ausländische Studierende besuchen die TH Wildau im Rahmen des DAAD und des SOKRATES/ERASMUS-Programms. So kamen zu Beginn des WS 2009/2010 von insgesamt 3954 Studierenden 8,7\% aus dem Ausland, in absoluten Zahlen 344 Studierende.[4] Ein Teil dieser Gäste wiederum kommt als Austauschstudierende aus Russland. Zudem studieren an der TH Wildau zahlreiche Russlanddeutsche als regulär eingeschriebene Studenten.[5]

\subsection{Veränderungen durch den Bologna-Prozess}

Die zunehmend internationale Ausrichtung von Hochschulen in den letzten Jahren und der Austausch von Studierenden vieler europäischer Länder untereinander [6] ist auch durch den so genannten Bologna-Prozess möglich, der sich zum Ziel setzt, das Zusammenwachsen Europas auch auf Hochschulebene zu fördern und die Mobilität europäischer Studierender innerhalb Europas zu erleichtern (Bologna-Erklärung 1999).[7] Auch an der TH Wildau sind in den letzten Jahren Studiengänge und Studieninhalte den Vorgaben des Bologna-Prozesses angepasst worden.

In der so genannten »Bologna-Erklärung « wurde 1999 in der italienischen Universitätsstadt Bologna die Absicht festgeschrieben, einen so genannten »Europäischen Hochschulraum « zu schaffen, der neben einem vergleichbaren Bachelor-Master-System ein europäisches System zur Anrechnung von Studienleistungen 
sowie die unkomplizierte Mobilität von Studierenden im Europäischen Hochschulraum ermöglichen soll, damit diese von Auslandsaufenthalten an Hochschulen außerhalb ihres Heimatlandes uneingeschränkt profitieren können (s. Bologna-Erklärung 1999 [8]).

\section{Stellenwert von Lehre und Lernen}

Als ein bedeutender Aspekt für den erfolgreichen Fortgang des Bologna-Prozesses wird die »Verbesserung von Lehre und Lernen « im europäischen Hochschulraum (s. Leuvener Kommuniqué 2009: 1 und 4, Adam 2010: 23, »Learning is not a spectator sport! « Önderoglu 2010: 23) sowie das Bestreben von »studierendenzentriertem Lernen « (Erklärung von Budapest und Wien zum Europäischen Hochschulraum 2010: 2) benannt. Letztere Zielsetzung wirft auch die Frage auf, wie es um die Orientierung auf die Studierenden in den einzelnen Ländern bestellt ist, was darunter zu verstehen sei und wie - auch aus interkultureller Perspektive - etwa in stärker hierarchisch orientierten Kulturen studierendenzentriertes Lernen in das kulturelle Verständnis integriert werden kann.

Bei aller Bereicherung eines Auslandssemesters oder -jahres beschreiben in der Tat viele Gaststudierende Lernverhalten, Lerngewohnheiten und Studienorganisation in Deutschland als von deren Heimatland erheblich abweichend. Auch Beobachtungen von Dozentinnen und Dozenten lassen vermuten, dass sich Studierende aus verschiedenen Ländern in den Hochschulveranstaltungen bezüglich ihrer Lerngewohnheiten anders verhalten als Studierende, die in Deutschland lernsozialisiert wurden.

Aufgrund dieser Beobachtungen wird im vorliegenden Beitrag die Frage gestellt, wie Studierende aus Russland ihr eigenes Lernverhalten im Kontext ihres Studiums in Deutschland einschätzen .

Studien und Untersuchungen der letzten Jahre zu diesem Thema zeigen, dass in der Tat Unterschiede im Lernverhalten, in der Lernsozialisation, im Lernstil etc. beobachtet werden (Barmeyer 2005; Bongards/ Stumpf/Leenen 2008; Queis 2009; Koptelzewa 2009). Anscheinend nicht immer fällt ausländischen Studierenden der Eingliederungsprozess hinsichtlich ihres Lernerfolges leicht. Oft scheint das Verstehen fremder Lernstrategien und die Adaption an diese sogar so viel Energie zu binden, dass gerade Austauschstudierende - wenn überhaupt - erst am Ende ihres Gastaufenthal- tes in Deutschland einen Lerngewinn erzielen können (s. Kap. 4).

In diesem Zusammenhang wird nun thematisiert, wie sich der Lernprozess aus Sicht der Betroffenen darstellt und welche Wünsche russische Studierende an der TH Wildau hinsichtlich einer besseren Unterstützung zur Erleichterung ihres Studienaufenthaltes seitens der Gasthochschule äußern. Daraus kann gefolgert werden, wie den aus Russland stammenden Studierenden im Studium bessere Unterstützung gewährt werden könnte, um vor allem den Lernprozess zu erleichtern und Lernerfolge sichern zu helfen.

\section{Umfrage unter Studierenden aus Russland}

Um möglichst authentische und konkrete Aussagen zum Thema »Lernsituation « treffen zu können, wurden 12 Studierende aus Russland zu ihren Eindrücken und Erfahrungen während ihres Studienaufenthalts an der TH Wildau schriftlich befragt.[9] Diese geringe Befragtenzahl kann natürlich nicht als repräsentativ gelten, ihre Meinung kann jedoch als Indikator für Aussagetendenzen gewertet werden. Im Folgenden sollen die Auswertung der Umfrage und ihre Ergebnisse vorgestellt werden. Es handelt sich dabei nicht um eine Evaluation der Lehrveranstaltungen seitens der Zielgruppe, sondern geht vielmehr darum, das Befinden der Studenten zu erfassen und sowohl positive Erfahrungen als auch mögliche Anpassungsschwierigkeiten während des Studienaufenthalts an einer deutschen Hochschule aus Sicht der Betroffenen darzustellen. Die gewonnenen Daten können als Anregung für weitere Überlegungen zur Optimierung von zukünftigen Studienaufenthalten von Austauschstudenten dienen.[10]

Die Informanden wurden zu mehreren Themenbereichen befragt. In der folgenden Teilauswertung wird der Schwerpunkt auf das Thema Lernsituation gelegt [11] Dabei liegt die Konzentration auf drei Aspekten:

- Beobachtungen während der Studienzeit an der TH Wildau

- eigene Schwierigkeiten

- Empfehlungen für das Auslandsstudium.

Die Studierenden konnten die auf Deutsch gestellten Fragen wahlweise auf Deutsch oder Russisch beantworten. 
3.1 Bewertung des Lehr- und Lernstils an der TH Wildau im Verhältnis zur Heimathochschule [12] Der »Lehrstil (ist) total anders als in meiner Universität« ist eine weitgehend übereinstimmende Aussage der Befragten der Gruppe der Austauschstudierenden und wird meist als attraktiv und interessant empfunden: »...материал предоставляется в максимально понятной форме: много наглядных материалов, презентации к лекциям и, конечно, практические занятия.« Das heißt: »Der Lernstoff wird in einer absolut verständlichen Form präsentiert: es wird viel veranschaulicht, die Vorlesungsfolien werden in schriftlicher Form zur Verfügung gestellt und vor allem gibt es praktische Übungen.«

Von allen Studierenden werden Professionalität der Dozenten und gute Vorbereitung sowie der Praxisbezug zum Beruf positiv hervorgehoben.

Hier sei angemerkt, dass die Studierenden aus Russland neben der Lernstoffverarbeitung auch noch eine mentale Umstellung vollziehen müssen. In ihrer Sozialisation als Studierende waren sie bis dato gewohnt, eher theoretischen Lernstoff aufzunehmen, sich einzuprägen und bei Bedarf zu reproduzieren. Zugespitzt wurde diese Studienkultur osteuropäischer Prägung von v. Queis (2009: 55) wie folgt beschrieben: »Denke nicht, sondern schreibe mit, lerne auswendig und bestehe die Prüfung«. Dagegen wird an deutschen Fachhochschulen theoretisches Wissen in Verknüpfung mit praktischen Vorgehensweisen »mit vielen Übungen und aktivem Experimentieren« (Bongards/ Stumpf/Leenen 2008: 213), d. h. in Anwendung dargeboten. Aktives Selbststudium wird dabei vorausgesetzt. Diese - offenbar für viele Studierende aus Russland neue - Lernsituation führt anfänglich meist zu Verunsicherung.

Auf die Frage danach, welchen Lehrstil die Studierenden bevorzugen, gibt es einen deutlichen Unterschied in den Antworten. Während Austauschstudierende zu drei Vierteln die Vorlesung bevorzugen, sehen länger in Deutschland Studierende aus Russland Vorteile in verschiedenen Lehrstilen: die jeweiligen Vorzüge sowohl von Vorlesungen als auch Einzel- und Gruppenarbeit bzw. Präsentationen werden mit Anschaulichkeit begründet und damit, dass Lernen in der Gruppe einprägsamer sei. »In den Vorlesungen erklären sie, nicht diktieren, deswegen konnten wir etwas verstehen, nicht nur schreiben.«Der Lernstoff würde anhand zahlreicher Beispiele erklärt.
In der Gruppenarbeit würden laut Befragtenangaben Studierende aus Russland auch durch Dozenten ermutigt, sich mit deutschen Studierenden zu mischen. So wird der Versuch unternommen, Sprachbarrieren durch den aktiv gesuchten Kontakt in der Gruppe zu überwinden und damit Sicherheit in der Fremdsprache als auch Lerninhalte und Selbstvertrauen zu stärken. Andere Befragte äußern allerdings sprachliche Schwierigkeiten bis hin zu Sprachbarrieren mit der Konsequenz, keinen Kontakt zu deutschen Studierenden aufbauen zu können. Sowohl der Lerneffekt als auch soziale Aspekte werden als Vorteile von Gruppenarbeit immer wieder hervorgehoben, auch Eigeninitiative werde bestärkt (»man ist gezwungen, selber etwas zu tun«). Als vorteilhaft wird auch die Erlangung sozialer Kompetenzen durch Gruppenarbeit und deren Bedeutung für die berufliche Nutzung erkannt.

Unterschiede im Lehr- und Lernstil im Verhältnis zur Heimatuniversität wurden auch in Zusammenhang mit der Atmosphäre und dem Kontakt zu Dozenten gebracht. Es herrsche eine »sehr entspannte Atmosphäre im Unterricht « und die »Dozenten waren besonders aufmerksam auf ausländische Studenten «.

\subsection{Aussagen zur Prüfungsform}

Im Hinblick auf Prüfungen äußerten alle befragten Austauschstudierenden, dass in Deutschland vorwiegend schriftliche Prüfungen durchgeführt würden, was für russische Studierende ungewohnt erscheint, da an russischen Hochschulen überwiegend mündlich geprüft wird. Gleichwohl wurde die schriftliche Form von einem Austauschstudierenden sogar als »die beste Form « bezeichnet, doch werden die Pausen zwischen den Prüfungen als zu kurz empfunden (»увеличить интервал для подготовки между экзаменами«), dies gleichermaßen von bereits länger an der TH Wildau Studierenden aus Russland, zumal die »Anzahl der Prüfungen doppelt so hoch« wie in Russland sei. Zudem wurden schriftliche Prüfungen als besonders schwer beschrieben, da »man mehr Zeit braucht, um die Aufgabe zu verstehen«.

Was die Unterrichtsmethodik betrifft, so scheint in der vorliegenden Situation die Umstellung der Betroffenen auf einen moderativen Lehrstil weniger schwierig ausgefallen zu sein als dies den Erhebungen (im geisteswissenschaftlichen Bereich) z. B. von Koptelzewa (2009) zu entnehmen ist. Dies könnte auch darauf zurückgeführt werden, dass in technischen und betriebswirtschaftlichen Studienrichtungen anders als in geisteswissenschaftlichen sowohl in Deutschland als 
auch in Russland eher ein "stark kognitiv ausgerichteter Lehrstil« (Barmeyer 2005: 183f.) herrscht. Folgerichtig hängen offenbar verschiedene Arbeitstechniken und Lehrmethodiken mit den zu vermittelnden Inhalten zusammen (ebd.).

Bei der hier befragten Zielgruppe handelt es sich wie oben erwähnt um Studierende technischer und betriebswirtschaftlicher Studiengänge. Es kann davon ausgegangen werden, dass die diesbezüglichen Lerninhalte im Unterschied zu geisteswissenschaftlichen Fächern stärker fakten- und materialbezogen angelegt und somit methodisch weniger prozessorientiert sind und kaum Meinungsbildung durch wissenschaftliche Diskurse erfordern. Infolgedessen wird in technisch bzw. wirtschaftlich ausgerichteten Lehrveranstaltungen verhältnismäßig weniger diskutiert als in geisteswissenschaftlichen. Auf diese fachliche und methodische Differenzierung ist möglicherweise zurückzuführen, dass die von ausländischen Studierenden sonst oft genannte Diskussionsfreudigkeit der deutschen Studierenden in der hiesigen Informandengruppe nicht einmal Erwähnung fand. In den Erhebungen von Koptelzewa (2009: 271ff.) hingegen stieß diese Art der Auseinandersetzung mit dem Lernstoff - nämlich Diskussion - bei russischen Studierenden auf deutliche Abneigung.

\section{Kulturelle Aspekte der Integration in das fremdkulturelle Lernumfeld}

In der einschlägigen Fachliteratur ist bereits wiederholt beschrieben worden, dass kulturelle Prägung bzw. bildungsbezogene Sozialisation von Studierenden das Lernverhalten auch in einer kulturell fremden Lernumgebung nachhaltig beeinflusst (Barmeyer 2005; Koptelzewa 2009; Rusch-Kurz 2000). Es besteht weitgehend Einigkeit darüber, dass ein Teil der Anpassungsschwierigkeiten während eines Studienaufenthalts in Deutschland kulturell bedingt ist. Dies betrifft nicht nur die kulturellen Unterschiede im Bereich der Alltagskommunikation, sondern auch die Abweichungen in den akademischen Bildungssystemen. Besonders deutlich scheint die Differenz zwischen »akademischen Kulturen« der osteuropäischen und der westeuropäischen Länder in den geisteswissenschaftlichen Fächern im Rahmen der universitären Lehre zu sein (Koptelzewa 2009, s. auch Kap. 2). So erführen die jeweiligen akademischen Traditionen sowohl durch Studierende als auch durch Dozierende auf beiden Seiten nicht ge- nügend gegenseitige Wertschätzung, die Unterschiede in den kommunikativen Strategien würden kaum wahrgenommen bzw. nicht richtig erkannt und dem Verhalten der jeweils anderen Seite in den Lehr- und Lernsituationen würde viel Defizitäres zugeschrieben, woran sich die "nostrozentrischen « Haltungen erkennen ließen (Koptelzewa 2009: 265ff.). Aus Sicht mittelund osteuropäischer Lernregionen unterstreicht Zgaga (2010) dies in seiner Rückschau auf die bisher erreichten Ziele im Bologna-Prozess, indem er »systemic barriers « benennt: »Europe's national systems have been traditionally different. «(Zgaga 2010: 7) und » ... system level incompatibilities between national frameworks existed « (ebd.) [13].

Für einen Teil der Anpassungsschwierigkeiten osteuropäischer Austauschstudenten werden oft die »autoritären Schulsysteme « verantwortlich gemacht, in denen die Betroffenen sozialisiert worden waren (Rusch-Kurz 2000: 85ff.). Das hierarchisch geprägte Verhalten setze sich auch an Hochschulen fort, »wo das Verhältnis [der Dozenten, R./S.] zu den Studenten durch Machtdemonstrationen und Distanz geprägt « sei (Thomas/ Yoosefi 2003: 34; vgl. auch Queis 2009: 55; Strewe 1994: $31)$.

Diese Feststellungen rekurrieren auf mindestens eine der vielzitierten Kulturdimensionen von Hofstede, die die russische und die deutsche Kultur voneinander unterscheiden: divergierender Grad der Machtdistanz [14]. Auch im Hochschulkontext erweist sich dieser für das kommunikative Verhalten in den Lernsituationen als wirksam (Koptelzewa 2009: 266ff.; Bongards/Stumpf/ Leenen 2008: 206f.).

Der kulturanthropologische Forschungsansatz, Kulturen über die zunächst unsichtbaren Wertesysteme mittels Beschreibungsgröße Kulturdimension »sichtbar« zu machen, hat sich als sehr fruchtbar erwiesen. Auf diesem Ansatz bauen viele spätere Untersuchungen über kulturelle Differenzen auf, die sich z. T. aus den Hofstede'schen Kulturdimensionen ergeben. So wurde im Rahmen der kulturvergleichenden Psychologie der relationale Begriff Kulturstandard (Thomas 2003: 119) als eine Beschreibungseinheit entwickelt, womit v. a. kulturspezifische Verhaltensmuster gemeint sind. Aus der Perspektive der deutschen Kultur wurden für die russische Kultur Kulturstandards wie Hierarchieorientierung, Kollektivorientierung, Personenorientierung u. a. ermittelt (Thomas/Yoosefi 2003). Kulturelle Aspekte der deutsch-russischen Zusammenarbeit bzw. die jeweiligen Besonderheiten des kommunikativen Ver- 
haltens auch auf anderen Feldern der Bildung wie auch in Geschäftsbeziehungen werden seit einigen Jahren in den Fachpublikationen eingehend beschrieben (Rösch 2005; Strewe 2005).

In der vorliegenden Datenerhebung sind vor allem diejenigen Äußerungen signifikant, die ein jeweils anderes Hierarchieverständnis belegen. So fiel den russischen Studierenden auf, dass die deutschen Lehrenden den Studierenden »nahe stehen « bzw. »gleichgestellt« seien, sich hilfsbereit zeigten (»Der Dozent ist ein Helfer für den Studenten «, »Sie diskutieren viel mit den Studenten«) und den Studierenden gegenüber »respektvoll, offen und freundlich« verhielten. Die Studenten würden hier in Bezug auf Anwesenheit, Hausaufgaben usw. weniger kontrolliert (»3а студентами меньше контроля«). Den Äußerungen war zu entnehmen, dass dies als positiv erlebt wurde. Andererseits beurteilten einige der Befragten das Verhalten von deutschen Studierenden, die die Nivellierung der Hierarchien (möglicherweise auch unbewusst) in Szene gesetzt hatten, als wenig respektvoll gegenüber den Dozenten.

\section{5 Überlegungen zur Optimierung der Lernsituation von Studenten aus Russland}

\subsection{Studienorganisation}

Der Hochschultyp Fachhochschule scheint den hier befragten Austauschstudierenden aus Russland in Bezug auf die Studienorganisation der technisch und betriebswirtschaftlich ausgerichteten Studiengänge um einiges vertrauter vorzukommen als die Studienorganisation an deutschen Universitäten, insbesondere in den geisteswissenschaftlichen Richtungen. Der Stundenplan wird in den Hochschulen (FH) im Großen und Ganzen vorgegeben. Fächerauswahl sowie Curriculum sind stringent aufgebaut, wodurch eine größere Ähnlichkeit zur Studienorganisation an den russischen Heimatuniversitäten entsteht. Im Unterschied zu deutschen Universitäten treffen russische (Austausch)Studierende an deutschen Fachhochschulen zudem eher auf geschlossene Seminargruppen, in denen die Studierenden über mehrere Semester zusammen lernen: sie kennen sich besser privat, organisieren sich in diversen Projektgruppen, fertigen Referate in Gruppen an und nehmen neben dem gemeinsamen Besuch von Vorlesungen zusammen an Laborübungen teil. Somit kommen gerade Austauschstudenten in eine "stehende»
Seminargruppe (mit schwach ausgeprägten dynamischen Gruppenbildungsprozessen). In dieser Hinsicht dürfte den russischen Studierenden die Integration in die Seminargruppen an einer deutschen Hochschule vom Typ Fachhochschule etwas leichter fallen als an einer Universität.

\subsection{Lehrstil}

Darüber hinaus scheint die Umstellung auf einen vorwiegend moderativen Lehrstil in nicht geisteswissenschaftlichen Fächern aufgrund der Fachspezifik in den Ingenieurwissenschaften bzw. der größeren Korrespondenz der Lehrinhalte wenig problematisch zu sein. Die stets positive Hervorhebung des Praxisbezuges in der Lehre durch die Befragten spricht dafür, dass das praxisorientierte Ausbildungskonzept einer Fachhochschule, welches sich im Einklang mit den hochschulpolitischen Rahmenvorgaben der Kultusministerkonferenz (»stärker anwendungsorientiert «) befindet (KMK 2003: 2), den Bedürfnissen der russischen Studierenden sehr entgegen kommt und eindeutig als Gewinn für die berufliche Tätigkeit verstanden wird.

\subsection{Wünsche und Empfehlungen der befragten Studierenden}

Wenngleich die Fachkompetenz und Professionalität der Lehrenden an der TH Wildau seitens der Befragten allgemein stark gewürdigt wurde, wurde in Bezug auf die Unterrichtsgestaltung doch eine andere eindringliche Botschaft an die deutschen Lehrenden artikuliert: den russischen Studierenden würde zugute kommen, wenn die Lehrenden dem zurückhaltenden Agieren der betroffenen Studierenden entgegenwirkten und die Integration der Betroffenen gezielt förderten, indem sie z. B. mehr Initiative ergriffen und die russischen Studierenden für Gruppenübungen auf die Seminargruppe »verteilten «, damit diese nicht »unter sich « blieben. Dieser Appell wird durch den Sachverständigenrat 2010, der in seinem Jahresbericht zur Integration ebenfalls auf die Bedeutung pädagogischer Öffnung hinweist (Sachverständigenrat 2010: 59), bestätigt.

Weiter äußerten die Befragten (sowohl die Austauschstudierenden als auch die bereits länger in Wildau Studierenden) ihre Schwierigkeiten mit der Organisation der Prüfungen: Angesichts der (etwas größeren als von zuhause gewohnten) Anzahl und des Schwierigkeitsgrades der Prüfungen wurde wiederholt beklagt, dass die Zeitintervalle zwischen den Prüfungsterminen enorm kurz seien. In Bezug auf die Durchführung der 
schriftlichen Prüfungen wurde mehrfach der Wunsch geäußert, die Aufgabenstellung von Dozenten mündlich erklärt zu bekommen, um sprachliche Missverständnisse auszuräumen.

Auf die Frage, was die Informanden ihren Kommilitonen in der Heimat empfehlen würden, wenn diese sich auf ein Studium in Deutschland vorbereiteten, wurde an erster Stelle mehrfach die Erhöhung der Sprachkompetenz im Deutschen betont (»Sie müssen sehr gut deutsch sprechen «). Dem folgten Empfehlungen, relevante Informationen über das Studium in Deutschland rechtzeitig einzuholen, außerdem Mahnungen, das »Studium in Deutschland ernst zu nehmen ", "Selbstständigkeit« zu entwickeln und sich auf Teamarbeit einzustellen bis hin zu einem Vorschlag, nicht alle gleichsprachigen Studenten zusammen in einem Wohnheim unterzubringen.

\subsection{Kulturelle Anpassung}

Um die oben genannten Anpassungsprozesse besser zu gestalten bzw. einen Kulturschock zu lindern, erachten die Autorinnen es als notwendig und hilfreich, den Austauschstudierenden sofort zu Beginn ihres Aufenthalts an jeder deutschen Hochschule ein Interkulturelles Training anzubieten bzw. sie in die stattfindenden Seminare für Interkulturelle Kommunikation einzubinden (Rösch 2010: 71ff.). Die Notwendigkeit einer solchen Maßnahme wurde bereits an vielen Hochschulen erkannt. Laut Kammhuber sei wichtig,

»selbständig und fortlaufend interkulturelle Lernproblematiken in Alltagssituationen wahrzunehmen, aktiv interkulturelles Wissen zu erschließen und Handlungsprobleme ... für das Wohlbefinden der eigenen Person, für die Beziehung zum Interaktionspartner und die Bewältigung der Aufgabe zu lösen sowie die dabei gewonnenen Erfahrungen bewerten und kommunizieren $\mathrm{zu}$ können.«(Kammhuber 2007: 13).

Auch Bildungs- und Zuwanderungsberichte heben immer wieder den hohen Stellenwert von zielgerichteten Bildungsmaßnahmen für den Integrationsprozess hervor (Sachverständigenrat 2010: 63).

\section{Fazit}

Abschließend kann man festhalten, dass Studierende aus Russland an der TH Wildau - so die Resultate der vorliegenden Umfrage - sich bemühen, mit den deutschen Hochschulgegebenheiten umzugehen, dabei etliche Unterschiede zum Heimatsystem feststellen und diese für sich teils positiv werten können. Allerdings benötigen sie offenbar auch viel Zeit und Energie dafür, dass sie sich erst in Deutschland Informationen zum deutschen Hochschulsystem und den Prüfungsgegebenheiten beschaffen bzw. Arbeitsweise und Lernstil aus eigener Erfahrung erleben können und somit in der ersten Zeit ihres Studienaufenthaltes viel Kraft in den Umstellungsprozess und die Adaptionsbemühungen investieren müssen.

Als weitere Maßnahme wäre daher zu empfehlen, bereits im Heimatland den Studierenden, die ins Ausland gehen möchten, entsprechende Vorbereitungstrainings, -seminare und Beratung durch ihre Auslandsämter, entsprechend geschulte Tutoren bzw. qualifiziertes Personal angedeihen zu lassen. Solche interkulturellen Trainings und ggf. auch Informationsveranstaltungen sollten speziell auf die Hochschul-, Lern- und Arbeitsgegebenheiten zwischen Heimat- und Zielland abgestimmt sein. Dies würde den mentalen und kulturellen Adaptionsprozess von Austauschstudierenden erheblich erleichtern, Kräfte für die inhaltliche Arbeit im Zielland freisetzen und damit den Erfolg eines Auslandsstudienaufenthaltes deutlich erhöhen können. 


\section{Anmerkungen}

1 Die diesem Artikel zugrunde liegenden Umfrageergebnisse werden in einer anderen thematischen Fassung zeitnah in der Online-Fachzeitschrift »Das Wort - Germanistisches Jahrbuch Russlands « veröffentlicht.

2 http://www.th-wildau.de/vor-dem-studium/hochschule/ geschichte.html - Abfrage am 21.07.2010.

3 Der Anteil ausländischer Studierender nahm deutlich zu: von 1,7 Prozent (im WS 2004/2005) auf 6,41 Prozent (im WS 2009/2010). (http://www.th-wildau.de/vor-dem-studium/hochschule/ portrait.html - 24.06.2010)

4 Im ersten Hochschulsemester im WS 2009/2010 beträgt diese Kennzahl 11,25 Prozent, im ersten Fachsemester im WS 2009/10 9,78 Prozent. (http://www.th-wildau.de/vor-dem-studium/ hochschule/portrait.html - Abfrage am 24.06.2010)

5 Diese sind quantitativ schwer zu benennen, da sie als Bürger mit deutschem Pass nicht statistisch geführt werden, häufig jedoch in Russland zur Schule gegangen, also dort lernsozialisiert sind.

6 Zuaktuellen Zahlen s.http://europa.eu/rapid/pressReleasesAction. do? reference $=$ STAT $/ 09 / 58 \&$ format $=$ HTML\&aged $=0 \&$ language $=\mathrm{D}$ E\&guiLanguage $=\mathrm{en}$

7 Für die Bundesrepublik Deutschland haben im Jahre 2006 12,8\% ausländischer Studierender die Gelegenheit wahrgenommen, für ein oder zwei Semester an einer deutschen Hochschule zu studieren (Daten der Bologna-Ministerkonferenz 2009).

8 Diese Erklärung geht wiederum auf die so genannte »Magna Charta Universitatum« von 1988 zurück, die hier nur erwähnt werden kann, s. hierzu die Entwicklung unter Bologna-Prozess 2010. Dem Abkommen gehören derzeit (2010) 47 Staaten an, darunter auch Deutschland und Russland (s. Erklärung von Budapest und Wien zum Europäischen Hochschulraum 2010: 1), die sich zum Ziel gesetzt haben, verbindliche Rahmen und geeignete Instrumente zur Umsetzung steigender und problemloserer Mobilität sowie zur Angleichung und gegenseitigen Anerkennung von Leistungen Studierender in Europa zu schaffen.

9 Von diesen 12 Studierenden gehören fünf Studierende in die Gruppe der Austauschstudierenden, die im WS 2009/10 für ein Semester an der TH Wildau studierten, sieben Studierende kommen ursprünglich aus Russland und studieren bereits seit mehr als einem Jahr an der TH Wildau.

10 Natürlich wäre eine größere Stichprobe wünschenswert, außerdem wäre auch eine Erhebung der Meinung von Dozenten mit gleicher Themenstellung von Nutzen. Diese konnten jedoch in diesem Rahmen nicht eingebracht werden.

11 Aufgrund der geringen Befragtenzahl wird mit einfachen Mehrheiten und Aussagetendenzen gearbeitet.

12 Als Heimathochschulen werden St. Petersburg und Jaroslavl genannt.

13 Auch das Londoner Kommuniqué von 2007 hält fest, dass Lernresultate für Studierende noch stärker in den Vordergrund gestellt werden müssen $(\S \S 2.2,2.4,3.5)$. In dem sich anschließenden Bologna-work program 2007-2009 heißt es dazu: »The aim is to develop a broader view of substantial differences, .... and, .... to seek to assess substantial differences in terms of learning outcomes. «(8)

14 Unter Machtdistanz wird »das Ausmaß, bis zu welchem die weniger mächtigen Mitglieder von Institutionen bzw. Organisationen eines Landes erwarten und akzeptieren, dass Macht ungleich verteilt ist « verstanden (Hofstede 2006: 59).

\section{Literatur}

Adam, St. (2010): Qualifications frameworks helping to reorient 21stcentury education. In: Ministry of Science and Research, Austria/ Ministry of Education and Culture, Hungary 2010: Bologna. 1999-2010.=duz_spec_Bologna.pdf, Abfrage am 11.06.2010. S. 23

Barmeyer, Ch. I. (2005): Interkulturelles Management und Lernstile. Studierende und Führungskräfte in Frankreich, Deutschland und Quebec. Campus Verlag Frankfurt/New York.

Bologna-Erklärung (1999) = Der Europäische Hochschulraum. Gemeinsame Erklärung der Europäischen Bildungsminister. 19. Juni 1999, Bologna. (Im Original: The European Higher Education Area. The Bologna Declaration of 19 June 1999. Joint declaration of the European Ministers of Education.)

Bologna-Ministerkonferenz (2009) = Bologna-Ministerkonferenz: 30\% der 25- bis 34-Jährigen in der EU27 absolvieren ein Hochschulstudium. Höherer Anteil an Frauen als Männern unter Hochschulabsolventen. Reference: STAT/09/58, Date: 28/04/2009 (http:// europa.eu/rapid/pressReleasesAction.do?reference=STAT/09/58\&f ormat $=$ HTML\&aged $=0 \&$ language $=$ DE\&guiLanguage $=$ en, Abfrage am 21.07.2010)

Bologna-Prozess $(2010)$ = Bologna-Prozess: Schaffung eines Europäischen Hochschulraums. Auf: http://europa.eu/legislation_summaries/education_training_youth/lifelong_learning/c11088_ de.htm, Abfrage am 21.07.2010

Bongards, M., Stumpf, S., Leenen, R. (2008): Interkulturelle Bezüge im Studium der Ingenieurwissenschaften - Erfahrungen und Konsequenzen am Campus Gummersbach der Fachhochschule Köln. In: Rösch, O. (Hrsg.): Technik und Kultur, Wildauer Schriftenreihe Interkulturelle Kommunikation, Band 6, Verlag News \& Media, Berlin.

Das Wort = DAAD/Schütz, St. (Hrsg.): Das Wort. Germanistisches Jahrbuch Russland. Moskauer Staatliche Universität (MGU), Moskau. Online: http://www.daad.ru/wort/wort.htm, Abfrage am 15.10.2010.

Harutyunyan, G., Bonete, R. (2010): Enhancing opportunities for students an staff. In: Ministry of Science and Research, Austria/ Ministry of Education and Culture, Hungary 2010: Bologna. 1999-2010.=duz_spec_Bologna.pdf, Abfrage am 11.06.2010, 31-32.

Hofstede, G. (2006): Lokales Denken, globales Handeln. Interkulturelle Zusammenarbeit und globales Management. 3. vollständig überarbeitete Auflage. Reihe Beck-Wirtschaftsberater, Deutscher Taschenbuch Verlag, München.

http://www.th-wildau.de/vor-dem-studium/hochschule/geschichte. html, Abfrage am 21.07.2010.

Kammhuber, St. (2007): Interkulturelles Lernen aus psychologischer Perspektive. Zur Qualitätssicherung interkultureller Trainings. In: Schmidt, Ch. M., Neuendorff, D. (Hrsg.): Sprache, Kultur und Zielgruppen. Bedingungsgrößen für die Kommunikationsgestaltung in der Wirtschaft, 9-26.

Key issues 2007 = Government Offices of Sweden (Hrsg.) (2007): Key issues for the European Higher Education Area - Social Dimension and Mobility. Västeras.

Koptelzewa, G. (2009): Interkulturalität in der akademischen Zusammenarbeit zwischen Ost und West. In: Umland, A., Bürgel, M. (Hrsg): Geistes- und sozialwissenschaftliche Hochschullehre in Osteuropa IV: Chancen und Hindernisse internationaler Bildungskooperationen. Peter Lang Verlag, Frankfurt. 
Leuvener Kommuniqué 2009 = Bologna-Prozess 2020. Der Europäische Hochschulraum im kommenden Jahrzehnt. Kommuniqué der Konferenz der für die Hochschulen zuständigen europäischen Ministerinnen und Minister, Leuven/Louvain-la-Neuve, 28. und 29. April 2009.

London Communiqué 2007 = Bologna Process. London Communiqué. Towards the European Higher Education Area: responding to challenges in a globalised world.

Önderoglu, S. (2010): Developing a National Qualifications Framework (NQF): The example of Turkey. In: Ministry of Science and Research, Austria/Ministry of Education and Culture, Hungary 2010: Bologna. 1999-2010. = duz_spec_Bologna.pdf, Abfrage am 11.06.2010, 23.

Queis, D. v. (2009): Interkulturelle Kompetenz. Praxis-Ratgeber zum Umgang mit internationalen Studierenden. WBG, Darmstadt.

Rösch, O. (2005): Gemeinsame Ziele - unterschiedliche Wege? Über die Gleichzeitigkeit des Ungleichzeitigen in der deutsch-russischen Zusammenarbeit, Veröffentlicht im September 2005 in der Internet-Zeitschrift für Kulturwissenschaften (INST). http://www. inst.at/trans/14Nr/roeschfv14.htm, Abfrage am 23.007.2010.

Rösch, O. (2010): Das Lehrgebiet Interkulturelle Kommunikation für Studierende der nicht-geisteswissenschaftlichen Studiengänge an der TFH Wildau. In: Eß, O. (Hrsg.): Das Andere lehren - Handbuch zur Lehre Interkultureller Handlungskompetenz, Münster, 71-83.

Rusch-Kurz, S. (2000): Interkulturelles Lernen in einem Akademischen Auslandsamt. In: Erdmann, K., Theisen, H. (Hrsg.): Der westöstliche Hörsaal. Interkulturelles Lernen zwischen Ost und West, Berlin, Wostok, 82-86.

Sachverständigenrat $2010=$ Sachverständigenrat deutscher Stiftungen für Integration und Migration $2010 \mathrm{Hg}$.: Einwanderungsgutachten 2010: Jahresgutachten 2010 mit Integrationsbarometer, Berlin.

Sekretariat der ständigen Konferenz der Kultusminister der Länder in der Bundesrepublik Deutschland (2003): 10 Thesen zur Bachelor- und Masterstruktur in Deutschland. Verfügbar unter: http://www.kmk.org/fileadmin/veroeffentlichungen beschluesse/2003/2003_06_12-10-Thesen-Bachelor-Master-in-D. pdf, Abfrage am 27.07.2010.

Strewe, B. (1994): Bildung in Russland. Vom allgemeinen Kulturgut zur privaten Investition. In: Grenzgänge - Kultur Ost, Kultur West. = DIE Zeitschrift für Erwachsenenbildung Nr. 2, 30-34.

Strewe, B. (2005): Eigenverantwortung und Selbstbestimmung. Erwachsenenbildung in Südosteuropa. In: Osteuropa. 55. Jg. 8/2005, 225-235.

Thomas, A. (2003): Kulturvergleichende Psychologie. 2., überarbeitete und erweiterte Auflage, Hogrefe, Göttingen/Bern/Toronto/Seattle.

Thomas, A., Yoosefi, T. (2003): Beruflich in Russland. Trainingsprogramm für Manager, Fach- und Führungskräfte. Vandenhoeck \& Ruprecht, Göttingen.

Zgaga, P. (2010): The Making of Bologna. In: Ministry of Science and Research, Austria / Ministry of Education and Culture, Hungary: Bologna 1999-2010. = duz_spec_Bologna.pdf, Abfrage am 11.06.2010. 7-10.

\section{Autorinnen}

Prof Dr. phil. Olga Rösch

Professur für Interkulturelle Kommunikation mit dem Schwerpunkt Mittel- und Osteuropa im Fachbereich Ingenieurwesen/Wirtschaftsingenieurwesen an der Technischen Hochschule Wildau [FH] Leitung des Instituts für Interkulturelle Kommunikation olga.roesch@th-wildau.de www.th-wildau.de/roesch

Bettina Strewe, Dr. phil.

Freiberufliche Dozentin für Kommunikation und Interkulturelle Kommunikation mit dem Schwerpunkt Mittel-, Südost- und Osteuropa an Hochschulen und in der Weiterbildung. InterKultOst Potsdam bettina.strewe@th-wildau.de www.bettina.strewe.de 